\title{
Lack of evidence that epidural pain relief during labour causes autism spectrum disorder: a position statement of the Canadian Anesthesiologists' Society
}

\section{Absence de preuve voulant que le recours à la péridurale pour soulager la douleur durant le travail cause le trouble du spectre de l'autisme}

\author{
Dolores M. McKeen, MD, MSc, FRCPC 두 - Valerie Zaphiratos, MD, MSc, FRCPC on \\ behalf of the Canadian Anesthesiologists' Society
}

Received: 17 October 2020/Accepted: 21 October 2020/Published online: 27 October 2020

(C) Canadian Anesthesiologists' Society 2020

Annually, millions of women worldwide give birth and benefit from epidural labour pain relief without complications. In Canada, over 360000 women gave birth and over $60 \%$ received epidural labour pain relief in 2018-19. ${ }^{1}$

The Canadian Anesthesiologists' Society (CAS) representing over 2,500 anesthesiologists, along with the CAS Obstetric Section, the Canadian Pediatric Anesthesia Society (CPAS), a sub-section of CAS, the Society for Obstetrics and Gynecology of Canada (SOGC), and the Canadian Paediatric Society (CPS) raise concerns with the findings of the study "Association Between Epidural Analgesia During Labor and Risk of Autism Spectrum Disorders in Offspring," published on October $12^{\text {th }}$ in JAMA Pediatrics. ${ }^{2}$ Those authors present a retrospective longitudinal database study of 147,895 children in a single American health system and conclude that there is an association between epidural pain relief in women delivering vaginally and subsequent autism spectrum disorder in their children. However, the true relationship between epidural pain relief and autism spectrum disorder has not been ascertained due to multiple methodological limitations in the study.

D. M. McKeen, MD, MSc, FRCPC ( $\square)$

IWK Women's \& Obstetric Department of Anesthesia,

Dalhousie University, Halifax, NS, Canada

e-mail: dolores.mckeen@iwk.nshealth.ca

V. Zaphiratos, MD, MSc, FRCPC

University of Montreal, Montreal, QC, Canada
The two study groups, women who received epidural pain relief and those who did not, were different in many ways and did not have the same health issues, socioeconomic status, or child characteristics. In addition, the details on pregnancy and delivery complications of these women, as well as other possible causes for autism, were not addressed in the article. No information is provided regarding the type and dose of the epidural medication administered. This article suggests potential danger associated with epidural analgesia without providing causal evidence. This could lead to potential harm and unnecessary guilt among pregnant women if they decide to opt for epidural pain relief, or in those whom epidural placement may be medically indicated to ensure the safety of both mother and baby.

The CAS, SOGC, and CPS reassure women, families, and health care providers that an association between labour epidural pain relief and autism spectrum disorder does not imply causation. Only randomized-controlled trials and their meta-analyses can confidently determine causation. Epidural labour analgesia is the most effective form of labour pain relief and remains the gold standard.

Over the last decade, research and innovative obstetric anesthesia care has led to advances that provide significant safety and other health benefits for women and babies. The desired outcome for labouring mothers is a positive experience for her and her family. The goal is to maintain the health of both mother and baby by preventing complications and allowing a rapid response to emergencies. Women contemplating epidural labour pain relief should consider the highest-level evidence: 
labour pain can be controlled, and satisfaction improved, with no increase in caesarean delivery rates. ${ }^{3}$

The CAS, SOGC and CPS agree with the UK and USA national specialty societies' position that support a woman's decision to choose epidural pain relief. ${ }^{4,5}$ This decision should take into consideration their personal needs, medical requirements, and cultural preferences, using the best evidence in careful discussion with their healthcare providers and anesthesiologists. Therefore, the recent study in JAMA Pediatrics should not alter current Canadian anesthetic care offered to obstetric patients. ${ }^{\mathrm{A}, \mathrm{B}}$

Chaque année, des millions de femmes partout dans le monde donnent naissance à un enfant et profitent d'une péridurale pour soulager la douleur durant le travail sans complications. En 2018-2019 au Canada, plus de 360000 femmes ont donné naissance à un enfant, et plus de $60 \%$ d'entre elles ont reçu une péridurale pour soulager la douleur durant le travail. ${ }^{1}$

La Société canadienne des anesthésiologistes (SCA), qui représente plus de 2500 anesthésiologistes, ainsi que la Section d'obstétrique de la SCA, la Société canadienne d'anesthésie pédiatrique (SCAP), une sous-section de la SCA, la Société canadienne de pédiatrie (SCP) et la Société des obstétriciens et gynécologues du Canada (SOGC) soulèvent des préoccupations concernant les conclusions de l'étude «Association Between Epidural Analgesia During Labor and Risk of Autism Spectrum Disorders in Offspring » publiée le 12 octobre dans JAMA Pediatrics. ${ }^{2}$ Les auteurs présentent une étude rétrospective et longitudinale d'une base de données de 147895 enfants d'un même système de santé américain et concluent à un lien entre la péridurale pour soulager la douleur chez les femmes qui accouchent par voie vaginale et un trouble du spectre de l'autisme subséquent chez leurs enfants. Toutefois, le véritable lien entre la péridurale pour soulager la douleur et le trouble du spectre de l'autisme ne peut être déterminé en raison des nombreuses limitations méthodologiques de l'étude.

Les deux groupes de l'étude, c'est-à-dire les femmes ayant reçu une péridurale pour soulager la douleur et celles n'ayant pas reçu de péridurale, étaient différents sous de nombreux aspects et ne présentaient pas les mêmes problèmes de santé, le même statut socioéconomique ou les mêmes caractéristiques concernant leurs enfants. En outre, les détails sur la grossesse et les complications à l'accouchement de ces femmes, ainsi que d'autres causes possibles de l'autisme, n'ont pas été abordés dans l'article. Aucun renseignement n'est fourni concernant le type et la

\footnotetext{
$\overline{\text { A CAS Executive Committee }}$ Approved 10.14.20.

в This position statement is available on the CAS website: https:// www.cas.ca/CASAssets/Documents/News/PosStat-EpiNoAut-EN.pdf
}

dose de la péridurale administrée. Cet article suggère un danger possible associé à l'analgésie péridurale sans fournir de preuve causale. Cette situation pourrait entraîner un éventuel préjudice et un sentiment de culpabilité injustifié chez les femmes enceintes qui décident de recevoir la péridurale pour soulager la douleur ou chez les femmes pour qui la péridurale peut être indiquée au plan médical pour assurer la sécurité de la mère et du bébé.

La SCA, la SCP et la SOGC rassurent les femmes, les familles et les fournisseurs de soins de santé sur le fait qu'un lien entre la péridurale pour soulager la douleur durant le travail et le trouble du spectre de l'autisme n'implique pas de causalité. Seuls des essais randomisés contrôlés et leurs méta-analyses peuvent déterminer la causalité avec certitude. L'analgésie péridurale durant le travail constitue le moyen le plus efficace de soulager la douleur durant le travail et demeure la référence absolue.

$\mathrm{Au}$ cours des dix dernières années, la recherche et les soins novateurs en anesthésie obstétricale ont mené à des avancées qui fournissent une sécurité considérable et d'autres avantages pour la santé des femmes et des bébés. Le résultat désiré pour les mères qui accouchent est une expérience positive pour elles et leur famille. L'objectif consiste à maintenir la santé de la mère et du bébé en prévenant des complications et en permettant de réagir rapidement aux situations d'urgence. Les femmes qui songent à la péridurale pour soulager la douleur durant le travail doivent tenir compte des données probantes du plus haut niveau : la douleur causée par le travail peut être contrôlée, et la satisfaction améliorée, sans augmentation du taux d'accouchements par césarienne. ${ }^{3}$

La SCA, la SCP et la SOGC sont en accord avec la position des sociétés nationales de spécialités du R.-U. et des États-Unis, qui soutiennent la décision d'une femme d'opter pour la péridurale pour soulager la douleur. ${ }^{4,5}$ Cette décision devrait tenir compte des besoins personnels des femmes, des exigences médicales et de leurs préférences culturelles, en se servant des meilleures données probantes dans le cadre d'une discussion approfondie avec leurs fournisseurs de soins de santé et anesthésiologistes. Par conséquent, l'étude récente parue dans JAMA Pediatrics ne devrait pas modifier les soins d'anesthésie actuellement offerts au Canada aux patientes en obstétrique. ${ }^{\mathrm{C}, \mathrm{D}}$

\footnotetext{
$\overline{\mathrm{C}}$ Comité directeur de la SCA Approuvé le 14 octobre 2020.

D Cet énoncé de position est disponible sur le site de la SCA au: https://www.cas.ca/CASAssets/Documents/News/PosStat-EpiNoAutFR.pdf
} 


\section{References}

1. Canadian Institute for Health Information (CIHI). Available from URL: https://apps.cihi.ca/mstrapp/asp/Main.aspx?Server=apmstrex tprd_i\&project=Quick\%20Stats\&uid=pce_pub_en\&pwd=\&evt=2048 $001 \&$ visualizationMode $=0 \&$ documentID $=029 \mathrm{DB} 170438205 \mathrm{AEBCC}$ 75B8673CCE822.

2. Qiu C, Lin JC, Shi JM, et al. Association Between Epidural Analgesia During Labor and Risk of Autism Spectrum Disorders in Offspring. JAMA Pediatr 2020; https://doi.org/10.1001/ jamapediatrics.2020.3231.
3. Anim-Somuah M, Smyth RMD, Cyna AM, Cuthbert A. Epidural versus non-epidural or no analgesia for pain management in labour. Cochrane Database Syst Rev 2018; 5: CD000331.

4. Royal College of Anaesthetists (RCoA). Available from URL: https://rcoa.ac.uk/news/no-evidence-labour-epidurals-cause-autism.

5. American Society of Anesthetists (ASA). Available from URL: https://soap.org/wp-content/uploads/2020/10/JAMAPeds_Epidurals_ SOAP_ASA_SPA_ACOG_SMFM_Media_Response_101220.pdf.

Publisher's Note Springer Nature remains neutral with regard to jurisdictional claims in published maps and institutional affiliations. 